\title{
TRATAMENTO DE LIXIVIADOS (CHORUME) UTILIZANDO A ÁGUA SUPERCRITICA
}

\author{
L. FERREIRA-PINTO ${ }^{1}$, J.M. FRANCO ${ }^{1}$, P.R.Z. DIAS ${ }^{1}$, J.C. LIMA ${ }^{1}$, A. F. ZANETTE ${ }^{1}$, C.R.G. \\ TAVARES $^{1}$; L. CARDOZO-FILHO ${ }^{1}$. \\ ${ }^{1}$ Universidade Estadual de Maringá, Departamento de Engenharia Química \\ E-mail para contato: leandrofpinto@gmail.com
}

\begin{abstract}
RESUMO - Este trabalho reporta dados experimentais da redução de DQO (Demanda Química de Oxigênio) através da oxidação de lixiviados (chorume) em água supercrítica em um reator de fluxo contínuo, construído em Inconel 625. As reações de oxidação foram realizadas na faixa de temperatura de $350-600{ }^{\circ} \mathrm{C}$, pressão de 15,0 e $22,5 \mathrm{MPa}$, concentração de DQO $1580 \mathrm{mg} . \mathrm{L}^{-1}$, fluxo de alimentação de 5 g.min ${ }^{-1}$ e tempo de reação de 40 minutos. A redução de DQO variou entre 50 - 98\%. A melhor condição de redução do DQO ocorreu na maior temperatura e na maior pressão $\left(600{ }^{\circ} \mathrm{C}\right.$ e $\left.22,5 \mathrm{MPa}\right)$ com redução de DQO de $\sim 98 \%$. Contudo, a $350{ }^{\circ} \mathrm{C}$ e $15,0 \mathrm{MPa}$ a redução do DQO foi de $\sim 55 \%$. Desta maneira, os resultados obtidos neste trabalho podem servir como base para estudos futuros da degradação de efluentes lixiviados (chorume) a partir da oxidação em água supercrítica.
\end{abstract}

\section{INTRODUÇÃO}

A oxidação/gaseificação em água supercrítica é um processo que ocorre a altas temperaturas e pressões na destruição eficiente de uma diversidade de resíduos orgânicos aquosos (Savage, 1999). Esse processo ocorre em condições acima do ponto crítico da água $\left(\mathrm{Tc} \geq 374{ }^{\circ} \mathrm{C}, \mathrm{Pc} \geq 22.1 \mathrm{MPa}\right)$ (Brunner, 2009). Sob estas condições, a água supercrítica apresenta propriedades físico-químicas únicas, que a tornam um meio eficaz para a oxidação de compostos orgânicos (García Jarana et al., 2008). Assim, as limitações de transporte de massa são minimizadas, levando a altas taxas de reação, com conversões próximas a $100 \%$ para tempos de residência curtos e reatores de pequeno porte (Abelleira et al., 2013; Veriansyah e Kim, 2007; Xu et al., 2012). Além disso, como as reações de oxidação são exotérmicas e endotérmicas, até mesmo a produção de calor pode ser alcançada neste processo (Abelleira et al., 2013; Bermejo et al., 2004; Cocero et al., 2002; Hellström et al., 2008; Jimenez-Espadafor et al., 2011; Silva et al., 2004; Smith and Wang, 2003). Diante destas características, e por não necessitar que os efluentes passem por tratamentos prévios sofisticados (Abelleira et al., 2013), a aplicação desta tecnologia torna-se um grande escape às tecnologias convencionais de tratamento de efluentes.

Efluentes provindos de águas residuais municipais (chorume), indústrias alimentícias (laticínios), indústrias químicas (corantes) são alguns dos efluentes que podem ser tratados a partir da oxidação/gaseificação em água supercrítica. Pois, a maioria desses efluentes é formada, basicamente por compostos orgânicos e água. Sendo, características excelentes para a aplicação desta técnica 


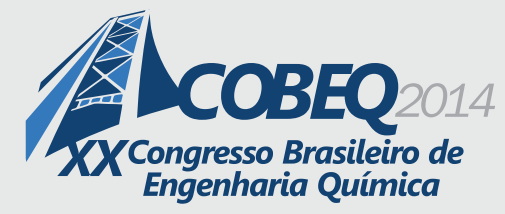

(Savage, 1999).

A motivação deste trabalho baseia-se na possibilidade da aplicação desta técnica de oxidação em água supercrítica no tratamento do chorume. Pois, o chorume é um efluente complexo com alta concentração de poluentes potencialmente adversos ao ambiente. Possui elevadas quantidades de matéria orgânica/inorgânica, substâncias húmicas, nitrogênio amoniacal, metais pesados, compostos orgânicos clorados e sais inorgânicos. Podendo contaminar águas subterrâneas e superficiais e contribuir na poluição do solo (Gong e Duan, 2010). Na maioria dos aterros, a forma de tratamento do chorume se dá por meio de processos biológicos - aeróbio (lodos ativados, lagoas aeradas e filtros biológicos) e anaeróbio (lagoas) (Zou et al., 2013). Entretanto, lançando mão somente do uso do tratamento biológico não é possível alcançar eficiência satisfatória na degradação da matéria orgânica (Wang et al., 2011). Além de apresentarem, em sua maioria, necessidade de grandes áreas e o inconveniente de gerar quantidades consideráveis de lodo. Por isso, faz-se necessário o estudo e a aplicação de técnicas inovadoras que possam permitir um tratamento com menor necessidade de área para sua implantação, menor tempo de processo e redução ou eliminação da geração de lodo. Métodos físico-químicos, como coagulação-flotação, precipitação-adsorção (Imai et al., 1998; Silva et al., 2004), oxidação químicas (Smith and Wang, 2003) e incineração são técnicas também aplicadas, no entanto, igualmente são insuficientes no tratamento completo deste efluente (Zou et al., 2013).

Neste sentido, a aplicação da oxidação em água supercrítica no tratamento de lixiviados torna-se uma tecnologia indispensável, visto o imenso potencial da água supercrítica na descontaminação de efluentes contendo resíduos orgânicos (Wang et al., 2011). Desta maneira, o objetivo deste trabalho é a degradação do chorume a partir de reações de oxidação, realizadas na faixa de temperatura de 400 $600{ }^{\circ} \mathrm{C}$, pressão de 15,0 e 22,5 MPa, concentração de DQO $\sim 1580 \mathrm{mg} . \mathrm{L}^{-1}$, fluxo de alimentação de 5 g.min ${ }^{-1}$ e tempo de reação de 40 minutos.

\section{EXPERIMENTAL}

\subsection{Materiais}

O chorume foi coletado em um aterro sanitário (Empresa Constroeste) da região de MaringáPR. Todos os reagentes químicos utilizados neste trabalho apresentam pureza analítica.

\subsection{Aparato e procedimento experimental}

O esquema do aparato experimental utilizado para produção de hidrogênio é apresentado na Figura 1, sendo composto por um reator de fluxo contínuo construído em Inconel 625, préaquecedor, condensador e tubulações, construídos em aço inoxidável 316. 


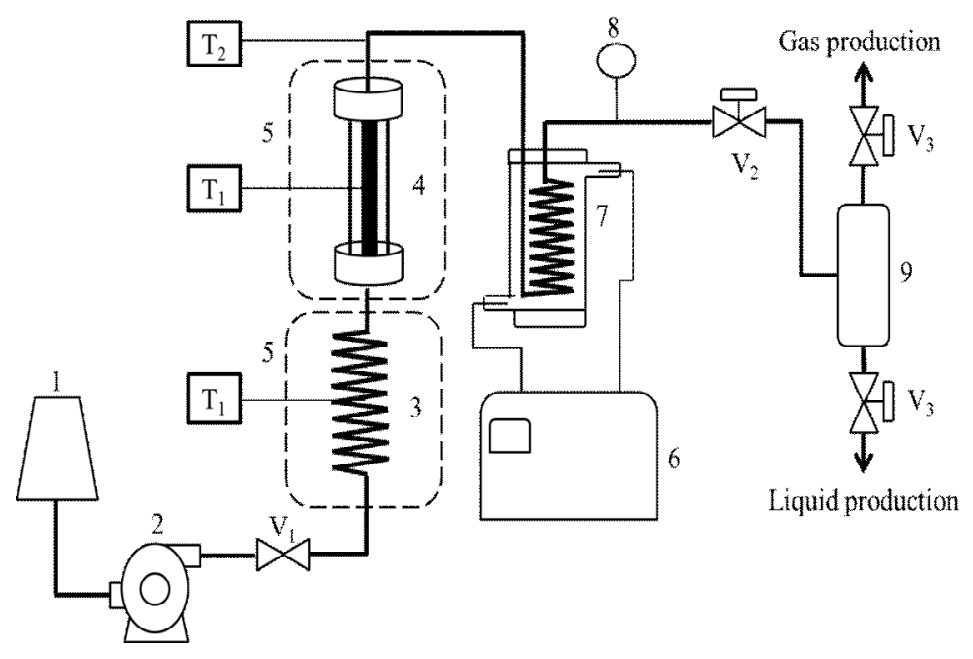

Figura 1 - Esquema do aparato de oxidação em água supercrítica. 1- Reservatório de alimentação;

2- Bomba de alta pressão; 3- Pré-aquecedor; 4- Reator; 5- Forno bipartido; 6- Banho termostatizado; 7- Condensador; 8- Manômetro; 9- Separador de fases; T1- Controlador de temperatura; T2- Indicador de temperatura; V1- Válvula de via-única; V2- Válvula Backpressure; e V3- Válvula.

O lixiviado contido em um reservatório de vidro (1) é succionada por meio de uma bomba de alta pressão, tipo HPLC (2). A mistura é transportada através de um tubo de aço inoxidável $316(L=1,5 \mathrm{~m}, \mathrm{I} . \mathrm{D} .=3,175 \mathrm{~mm})$ até a válvula $\mathrm{V}_{1}$ (válvula de via única) e em seguida distribuída até o pré-aquecedor (3), $(L=6 \mathrm{~m}$, I.D. $3,175 \mathrm{~mm})$. A mistura reacional, devidamente aquecida $\left(50^{\circ} \mathrm{C}\right)$, entra no reator $(4)(L=0.2 \mathrm{~m}$, I.D. $=13 \mathrm{~mm})$. O aquecimento do reator e do préaquecedor é realizado através de dois fornos bipartidos (5), equipados, cada um, com duas resistências infravermelhas em cerâmicas (Corel-Brazil) com potência de $1000 \mathrm{~W}$. O acionamento das resistências é realizado por controladores (Novus-Brazil), conectados aos termopares do tipo $\mathrm{J}$ (precisão $\left.\pm 1,0{ }^{\circ} \mathrm{C}\right)\left(\mathrm{T}_{1}\right)$ em contato ao pré-aquecedor e reator. Após percorrer o reator, a mistura resultante segue por um tubo de aço inoxidável $316(\mathrm{~L}=2,5 \mathrm{~m}$, I.D. $=3,175 \mathrm{~mm}$ ) para troca de calor com o ambiente externo, com monitoramento da temperatura realizado por um indicador de temperatura da (Novus-Brazil), conectado a um termopar tipo T (precisão $\left.\pm 1.0^{\circ} \mathrm{C}\right)\left(\mathrm{T}_{2}\right)$. Em seguida, a mistura resultante entra no condensador (7), uma serpentina de aço inoxidável $316(\mathrm{~L}=2,5 \mathrm{~m}, \mathrm{I} . \mathrm{D} .=3,175 \mathrm{~mm})$, para resfriamento à temperatura de $10{ }^{\circ} \mathrm{C}$. Após, a mistura resultante chega à válvula back-pressure $\left(\mathrm{V}_{2}\right)$, para ajuste da pressão, observado no manômetro (8). No coletor/separador de fases (9) ocorre a separação entre a fase líquida e vapor. A fase líquida é composta por lixiviados reagido e não reagido e outros produtos formados na reação e a fase vapor composta pelos produtos gasosos da reação, principalmente por, $\mathrm{H}_{2}, \mathrm{CO}, \mathrm{CO}_{2}$ e $\mathrm{CH}_{4}$. 


\subsection{Análises}

A concentração de carbono orgânico total foi obtida pela diferença entre a concentração de carbono total e a de carbono inorgânico. As amostras foram filtradas em membrana $0,45 \mu \mathrm{m}$ e acidificadas até $\mathrm{pH} 2$ e armazenadas a $4^{\circ} \mathrm{C}$, para posterior análise em um analisador de carbono, marca Shimadzu - Total Organic Carbon Analyzer - modelo TOC-L. A redução do COT será calculada de acordo com a equação 1 .

$$
R e d u c ̧ a \tilde{a}{ }_{-} T O C(\%)=\frac{T O C_{\text {entrada }}-T O C_{\text {saida }}}{T O C_{\text {entrada }}} \times 100
$$

\section{RESULTADOS E DISCUSSÃO}

Para verificar a confiabilidade do aparato e procedimento experimental proposto neste projeto, foram realizadas reações de gaseificação do etanol a $550{ }^{\circ} \mathrm{C}$ e $600{ }^{\circ} \mathrm{C}$ para uma razão molar $\mathrm{H}_{2} \mathrm{O}: \mathrm{EtOH}$ [20:1] (Figura 2) e comparados com dados experimentais obtidos por Therdthianwong et al. (2011). Os dados de conversão do etanol em gases apresentaram boa concordância com os dados reportados da literatura.
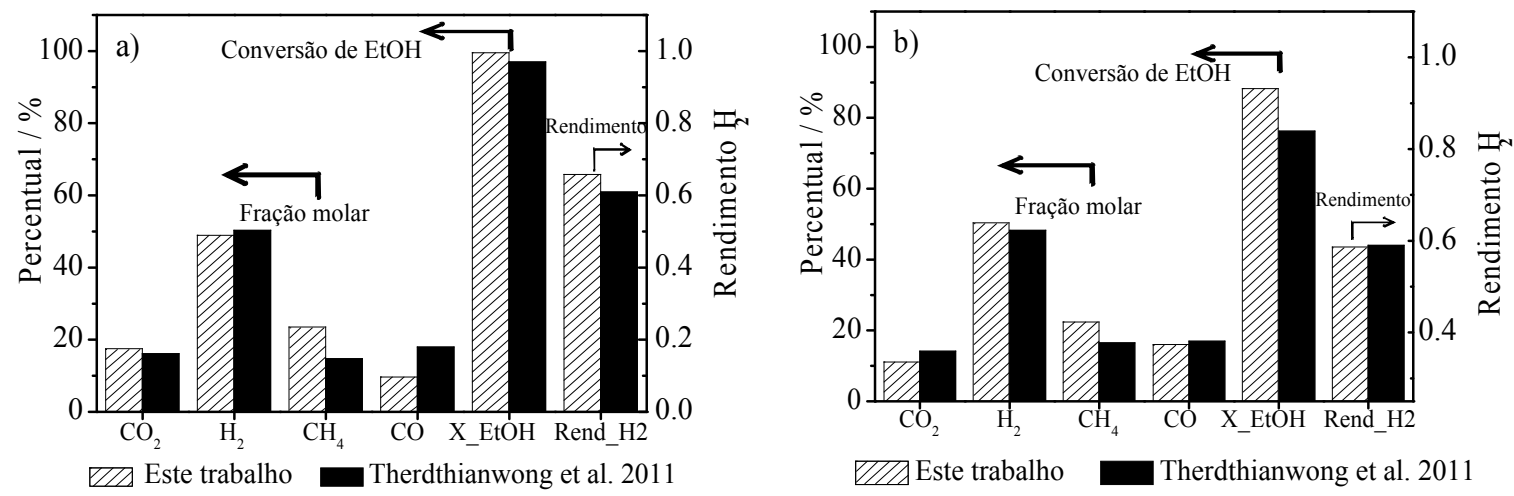

Figura 2. Dados experimentais de fração molar dos gases formados, conversão de EtOH e rendimento de $\mathrm{H}_{2}$ a partir da gaseificação em água supercrítica da lactose [razão molar H2O:EtOH (20:1), 25,0 MPa] (a) $550^{\circ} \mathrm{C}$ e (b) $600{ }^{\circ} \mathrm{C}$, obtidos neste estudo e reportado do trabalho de Therdthianwong et al. 2011.

Após validação da metodologia experimental foram realizadas reações de oxidação de águas residuais municipais (chorume) em água supercrítica. As reações de oxidação foram realizadas na faixa de temperatura de $350-600^{\circ} \mathrm{C}$, pressão de 15,0 e $22,5 \mathrm{MPa}$, concentração inicial de DQO $\sim 1580 \mathrm{mg} / \mathrm{L}$, fluxo de alimentação de 5 g. $\min ^{-1}$, tempo de residência de $\sim 30$ segundos e tempo de reação de 40 minutos. A redução de DQO variou entre $\sim 50$ - 98\% (Tabela $1)$. 
Tabela 1. Dados experimentais de degradação de lixiviado (chorume).

\begin{tabular}{ccccc}
\hline \multirow{3}{*}{ Temperatura ${ }^{\circ} \mathrm{C}$} & $15 \mathrm{MPa}$ & $15 \mathrm{MPa}$ & $22,5 \mathrm{MPa}$ & $22,5 \mathrm{MPa}$ \\
\cline { 2 - 5 } & $\begin{array}{c}\text { Concentração de } \\
\text { DQO }\end{array}$ & $\begin{array}{c}\text { Remoção de } \\
\text { DQO }\end{array}$ & $\begin{array}{c}\text { Concentração de } \\
\text { DQO }\end{array}$ & $\begin{array}{c}\text { Remoção de } \\
\text { DQO }\end{array}$ \\
\hline 350 & 731.3 & $53.7 \%$ & 561 & $64.5 \%$ \\
400 & 787.8 & $50.1 \%$ & 517.5 & $67.2 \%$ \\
500 & 610.7 & $61.3 \%$ & 227 & $85.6 \%$ \\
600 & 218 & $86.2 \%$ & 39 & $97.5 \%$ \\
\hline
\end{tabular}

A melhor condição de redução do DQO ocorreu na maior temperatura e na maior pressão $\left(600{ }^{\circ} \mathrm{C}\right.$ e $\left.22,5 \mathrm{MPa}\right)$ com redução de $\mathrm{DQO}$ de $\sim 98 \%$. Contudo, a $350{ }^{\circ} \mathrm{C}$ e $15,0 \mathrm{MPa}$ a redução do DQO foi de $\sim 50 \%$. Na Figura 2 observa-se o efeito da pressão e da temperatura na degradação do lixiviado (chorume). Independente da pressão, a elevação da temperatura é um fator chave na redução da DQO. No caso da pressão, a elevação da pressão de $15 \mathrm{MPa}$ para $22.5 \mathrm{MPa}$ aumentou a eficiência da degradação em aproximadamente $20-30 \%$.

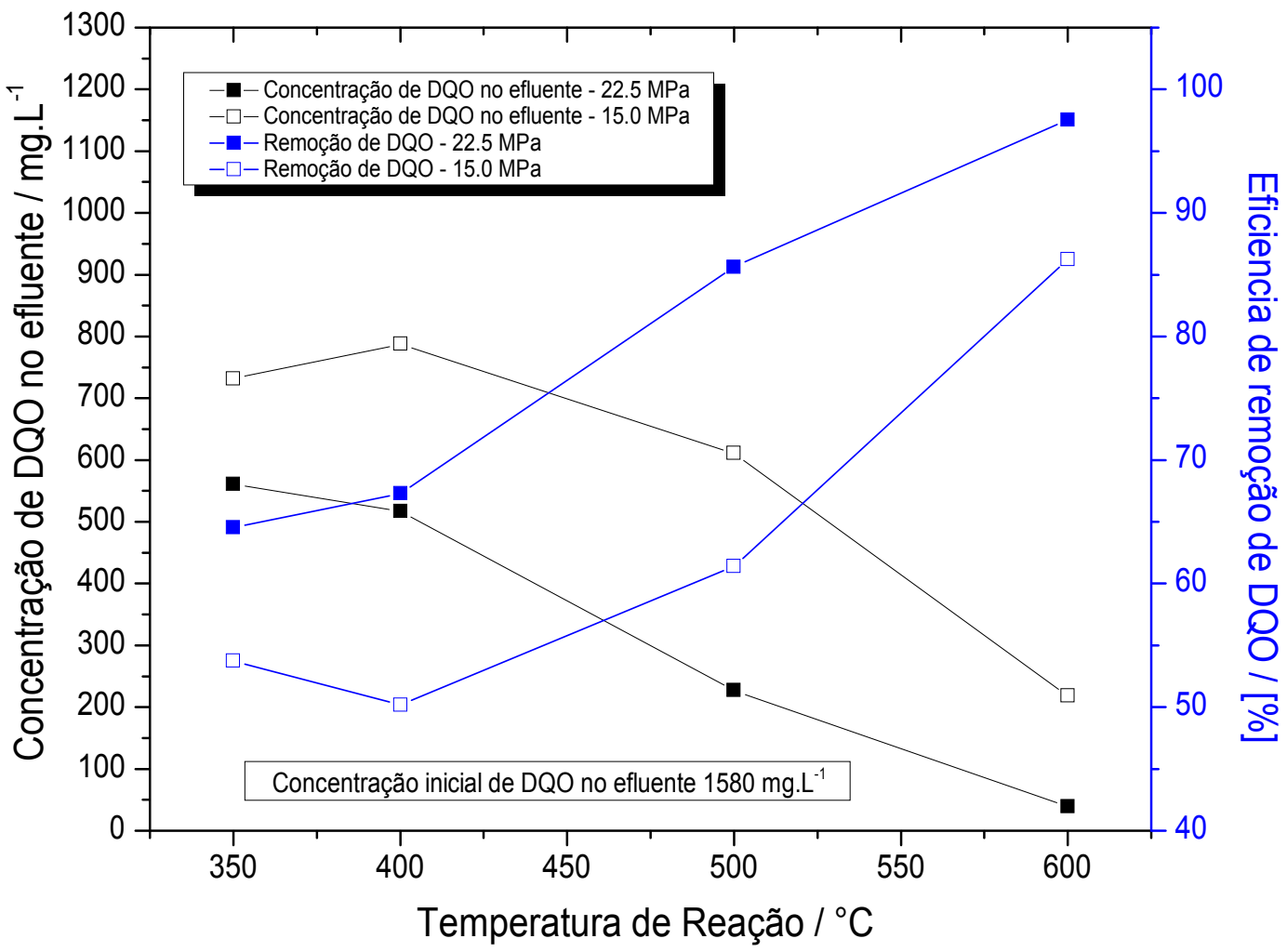

Figura 2. Dados experimentais de redução de DQO obtidos nas pressões de $15 \mathrm{MPa}$ e 22,5 MPa. 
A partir destes dados de redução de DQO do chorume, onde observa-se uma redução de aproximadamente $98 \%$, verifica-se que a utilização da oxidação em meio com água supercrítica é uma promissora técnica de tratamento de efluentes.

\section{CONCLUSÃO}

Este trabalho teve como proposta estudar a oxidação de lixiviado (chorume) em água supercrítica. Onde, verificou-se que a elevação da temperatura foi um fator primordial na redução eficiente do TOC, pois, em reações a $350{ }^{\circ} \mathrm{C}$ e $15 \mathrm{MPa}$ a redução do TOC foi de aproximadamente $55 \%$, enquanto que, a $600{ }^{\circ} \mathrm{C}$ e $22,5 \mathrm{MPa}$ a redução foi próxima aos $100 \%$. Desta maneira, verifica-se que os dados obtidos neste trabalho podem servir como base inicial para futuros estudos que envolvam efluentes de lixiviados (chorume). Pois, verificou-se que a metodologia de oxidação supercrítica deste efluente apresentou resultados interessantes de redução de TOC, sendo uma alternativa considerável no tratamento de efluentes.

\section{AGRADECIMENTOS}

Agradecemos aos suportes financeiros concedidos pelo CNPq e Fundação Araucária/Paraná.

\section{REFERÊNCIAS}

Abelleira, J., Sánchez-Oneto, J., Portela, J.R., Martínez de la Ossa, E.J., 2013. Kinetics of supercritical water oxidation of isopropanol as an auxiliary fuel and co-fuel. Fuel 111, 574 583. doi:10.1016/j.fuel.2013.03.033

Bermejo, M.., Cocero, M.., Fernández-Polanco, F., 2004. A process for generating power from the oxidation of coal in supercritical water. Fuel 83, 195-204. doi:10.1016/S00162361(03)00256-4

Brunner, G., 2009. Near and supercritical water. Part II: Oxidative processes. J. Supercrit. Fluids 47, 373-381. doi:10.1016/j.supflu.2008.09.001

Cocero, M.., Alonso, E., Sanz, M.., Fdz-Polanco, F., 2002. Supercritical water oxidation process under energetically self-sufficient operation. J. Supercrit. Fluids 24, 37-46. doi:10.1016/S0896-8446(02)00011-6

García Jarana, M.B., Sánchez-Oneto, J., Portela, J.R., Nebot Sanz, E., Martínez de la Ossa, E.J., 2008. Supercritical water gasification of industrial organic wastes. J. Supercrit. Fluids 46, 329-334. doi:10.1016/j.supflu.2008.03.002

Gong, W., Duan, X., 2010. Degradation of landfill leachate using transpiring-wall supercritical water oxidation (SCWO) reactor. Waste Manag. 30, 2103-7. doi:10.1016/j.wasman.2010.04.028

Hellström, D., Baky, A., Jeppsson, U., Jönsson, H., Kärrman, E., 2008. Comparison of Environmental Effects and Resource Consumption for Different Wastewater and Organic 
Waste Management Systems in a New City Area in Sweden. Water Environ. Res. 80, 708 718. doi:10.2175/106143008X276705

Imai, A., Onuma, K., Inamori, Y., Sudo, R., 1998. Effects of Pre-Ozonation in Refractory Leachate Treatment by the Biological Activated Carbon Fluidized Bed Process. Environ. Technol. 19, 213-221. doi:10.1080/09593331908616673

Jimenez-Espadafor, F., Portela, J.R., Vadillo, V., Sánchez-Oneto, J., Becerra Villanueva, J.A., Torres García, M., Martínez de la Ossa, E.J., 2011. Supercritical Water Oxidation of Oily Wastes at Pilot Plant: Simulation for Energy Recovery. Ind. Eng. Chem. Res. 50, 775-784. doi:10.1021/ie101166j

Savage, P.E., 1999. Organic Chemical Reactions in Supercritical Water. Chem. Rev. 99, 603622.

Silva, A.C., Dezotti, M., Sant'Anna, G.L., 2004. Treatment and detoxification of a sanitary landfill leachate. Chemosphere 55, 207-14. doi:10.1016/j.chemosphere.2003.10.013

Smith, D.W., Wang, F., 2003. Application of advanced oxidation methods for landfill leachate treatment - a review. J. Environ. Eng. Sci. 2, 413-427. doi:10.1139/s03-058

Therdthianwong, S., Srisiriwat, N., Therdthianwong, A., Croiset, E., 2011. Hydrogen production from bioethanol reforming in supercritical water. J. Supercrit. Fluids 57, 58-65. doi:10.1016/j.supflu.2011.02.005

VERIANSYAH, B., KIM, J.-D., 2007. RETRACTED: Supercritical water oxidation for the destruction of toxic organic wastewaters: A review. J. Environ. Sci. 19, 513-522. doi:10.1016/S1001-0742(07)60086-2

Wang, S., Guo, Y., Chen, C., Zhang, J., Gong, Y., Wang, Y., 2011. Supercritical water oxidation of landfill leachate. Waste Manag. 31, 2027-35. doi:10.1016/j.wasman.2011.05.006

Xu, D., Wang, S., Tang, X., Gong, Y., Guo, Y., Wang, Y., Zhang, J., 2012. Design of the first pilot scale plant of China for supercritical water oxidation of sewage sludge. Chem. Eng. Res. Des. 90, 288-297. doi:10.1016/j.cherd.2011.06.013

Zou, D., Chi, Y., Fu, C., Dong, J., Wang, F., Ni, M., 2013. Co-destruction of organic pollutants in municipal solid waste leachate and dioxins in fly ash under supercritical water using $\mathrm{H} 2 \mathrm{O} 2$ as oxidant. J. Hazard. Mater. 248-249, 177-84. doi:10.1016/j.jhazmat.2013.01.005 